\title{
Damage Localization Based on Symbolic Time Series Analysis
}

\author{
Mehrisadat Makki Alamdari ${ }^{*}$, Bijan Samali, Jianchun Li \\ *Corresponding author:mehri.makki@gmail.com
}

Centre for Built Infrastructure Research, University of Technology, Sydney, 15 Broadway Ultimo NSW, Australia

\begin{abstract}
The objective of this paper is to localize damage in a single or multiple state at early stages of development based on the principles of symbolic dynamics. Symbolic Time Series Analysis (STSA) of noise-contaminated responses is used for feature extraction to detect and localize a gradually evolving deterioration in the structure according to the changes in the statistical behavior of symbol sequences. Basically, in STSA, statistical features of the symbol sequence can be used to describe the dynamic status of the system. Symbolic dynamics has some useful characteristics making it highly demanded for implementation in real-time observation application such as structural health monitoring. First, it significantly reduces the dimension of information and provides information-rich representation of the underlying data. Second, symbolic dynamics and the set of statistical measures built upon it represent a solid framework to address the main challenges of the analysis of nonstationary time data. Finally, STSA often allows capturing the main features of the underlying system while alleviating the effects of harmful noise. The method presented in this paper consists of four primary steps: (i) acquisition of the time series data; (ii) creating the symbol space to produce symbol sequences based on the wavelet transformed version of time series data; (iii) developing the symbol probability vectors to achieve anomaly measures; (iv) localizing damage based on any sudden variation in anomaly measure of different locations. The method was applied on a flexural beam and a 2-D planar truss bridge subjected to varying Gaussian excitation in presence of $2 \%$ white noise to examine the efficiency and limitations of the method. Simulation results under various damage conditions confirmed the efficiency of the proposed approach for localization of gradually evolving deterioration in the structure, however, for the future work the method needs to be verified by experimental data.
\end{abstract}

Keywords: Damage Localization, Symbolic Dynamics, Probability Vector, Symbol Space, Wavelet Coefficients, Measurement Noise.

\begin{tabular}{|c|c|c|c|}
\hline \multicolumn{4}{|l|}{ Nomenclature } \\
\hline$\left\{x_{t}^{j}\right\}$ & Response of the structure in $j$-th sensor at date $t$ & $p\left(\lambda_{i}\right)$ & Probability of occurrence of symbol $\lambda_{i}$ \\
\hline$X^{j}=\left[\left\{x_{0}^{j}\right\},\left\{x_{1}^{j}\right\},\left\{x_{2}^{j}\right\}, . .,\left\{x_{T}^{j}\right\}\right]_{N \times T}$ & All measured responses in $j$-th sensor & $H(S)$ & Shannon entropy \\
\hline$\Omega$ & State space & $\left\{\widetilde{y}_{t}^{j}\right\}$ & Normalized response in $j$-th sensor at date $t$ \\
\hline$\Lambda$ & Symbol space & $\left\{\widetilde{y}_{0}^{j}\right\}$ & $\begin{array}{l}\text { Normalized response in } j \text {-th sensor at initial stage } \\
\text { of observation }\end{array}$ \\
\hline$S$ & Number of partitions & $\sigma_{t}^{j}$ & $\begin{array}{l}\text { Standard deviation of the observed signal in } j \text {-th } \\
\text { sensor at date } t\end{array}$ \\
\hline$T$ & Number of monitoring stages & $M$ & Number of sensors \\
\hline$N$ & The length of the response signal & $\zeta$ & Threshold value during DWT process \\
\hline$\Delta_{t}$ & Location of maximum anomaly measure at stage $t$ & $\Psi$ & Mapping from state space to symbol space \\
\hline $\mathbf{P}_{t}^{\mathrm{j}}$ & Probability vector in $j$-th sensor at date $t$ & $\lambda_{i}$ & The $i$-th partition in the symbol space \\
\hline$\left\{\bar{\varepsilon}_{t}^{j}\right\}$ & $\begin{array}{l}\text { Deterministic time response of strain in } j \text {-th sensor } \\
\text { at date } t\end{array}$ & $d$ & Original detail coefficient \\
\hline$\mu_{t}^{j}$ & $\begin{array}{l}\text { Mean value of the observed signal in } j \text {-th sensor at } \\
\text { date } t\end{array}$ & $\delta$ & Recovered detail coefficient \\
\hline$A_{t}^{j}$ & Anomaly measure in $j$-th sensor at date $t$ & $R(t)$ & A series of random data to create random noise \\
\hline$\left\{\bar{u}_{t}^{a}\right\}$ & $\begin{array}{l}\text { Deterministic recorded time response in node } \\
\text { number } a \text {, at date } \mathrm{t} \text {, in } \mathrm{x} \text { direction }\end{array}$ & $\left\{\bar{v}_{t}^{a}\right\}$ & $\begin{array}{l}\text { Deterministic recorded time response in node } \\
\text { number } a, \text { at date } \mathrm{t}, \text { in y direction }\end{array}$ \\
\hline$h(S)$ & Dynamic entropy & & \\
\hline
\end{tabular}




\section{Introduction}

Early detection of structural damage in infrastructures is crucial to avoid life and economic losses due to catastrophic failures. In this regard, structural health monitoring (SHM) evaluates the integrity of a structure by observing its dynamic responses by an array of sensors over time to determine the current health state of the structure. One of the major obstacles facing SHM is the analysis of huge amount of data that is produced. Therefore, it is important to obtain more compact representation of the data with no significant loss of information. On this point, feature extraction as a data transform technique can be applied to transform the input data into a set of features to reduce the dimension of information and provide information-rich representation of the underlying data.

Conventional vibration-based feature extraction methods are separated into two categories; the first group includes methods utilizing Fourier analysis to extract damage-indicative features such as Frequency Response Functions (FRF) and modal parameters (natural frequencies, mode shapes, modal damping) [1,2]. Fourier analysis is sufficient once the system's behavior is stationary (the system's statistical parameters are constant). If the statistical properties of the system under study vary over time, consistent comparison of the system will not be possible. On this point, the second category is comprised of methods dealing with non-stationary signals with frequency-time analysis approach such as Short Time Fourier Transform (STFT) [3, 4], wavelet analysis [5-7] and Hilbert-Huang Transformation (HHT) $[8,9]$.

Recently, several new statistical based feature extraction approaches have been proposed for structural health monitoring. Statistical pattern recognition methods such as Principal Component Analysis (PCA) [10-13], Independent Component Analysis (ICA) [14-16] and neural network-based techniques are regarded as useful methods for feature extraction of time-series data [17-20].

Traditional feature extraction methods are inadequate in characterization of high-dimensional and non-linear systems. A novel scheme of symbolic dynamics has recently been introduced for feature extraction of time series data in several applications [21]. Symbolic dynamics studies long term evolution of dynamical systems with preserving and highlighting key features of the history which is useful for SHM to detect any gradually evolving deterioration in the structure [22]. In a recent study the performance of STSA has been shown to be superior to that of several pattern classification techniques such as principal component analysis (PCA), artificial neural networks (ANN) and kernel regression analysis (KRA), in terms of early detection of changes and computation efficiency [23].

In STSA, basically, there is no prior assumption about the nature of the system under study as the method can be applied for deterministic or stochastic, linear or non-linear systems. In symbolic time series analysis, the behavior of a system, linear or non-linear, deterministic or stochastic is represented via symbols in a constructed space. The underlying concept in the technique is to describe the raw time-series measurements with a corresponding sequence of symbols. Symbolization is treated as a transform from state space into symbol space which significantly reduces numerical computations in the subsequent analysis while retaining important information, which is vital for real-time monitoring applications. One of the effectiveness accompanying the technique is that symbolic encoding applied to times series data often improves signal-to-noise ratio, hence, feature extraction by conducting this approach is less sensitive to measurement noise [24-28].

In this study, symbolic dynamics of time series data has been developed and analyzed to detect and localize a gradually evolving change in a system. In section 2, first a background on the topic of STSA will be presented followed by the damage localization algorithm in section 3 . The feasibility of the proposed method on localization of damage is examined via extensive numerical simulations with various damage conditions in section 4 . The numerical simulation consists of a flexural beam and a 2D planar truss bridge subjected to gradual deterioration. Section 5 concludes the work and suggests some future works. 


\section{A background on Symbolic Time Series Analysis (STSA)}

Symbolic dynamics is application of information theory to dynamical systems. It is a powerful data analysis and data transform technique for studying behavior of continuously varying dynamical systems which considerably reduces the dimension of the problem. A central step in the technique is to discretize the raw time-series measurements into a corresponding sequence of symbols. The idea is to partition the range of original observations into a finite number of cells (subsets or partitions) called generating partitions. Each partition is associated with one distinct symbol and then the original time data is uniquely mapped into symbol space based on the range in which each value in the measurement lies. After creating the symbol sequence, the statistical pattern of the symbol sequence is investigated to evaluate the behavior of the dynamical system and also to detect any possible change in the system parameters. One great advantage accompanying the technique lies in the fact that the efficiency of numerical computation is considerably increased over what it would be for the original data; this is particularly beneficial for real-time monitoring.

STSA allows capturing the main features of the underlying system while alleviating the effects of harmful noise. The effectiveness of STSA in noisy condition has been demonstrated in several research [25-28]. The results of research presented in [25], show that compared to artificial neural network-based method (ANN) and support vector machine-based method (SVM), STSA-based approach provides more robust damage indices in presence of measurement noise. In another research, it has been indicated that symbolization can reduce sensitivity to measurement noise [26]. In a different study, it has been shown that symbolization can directly enhance signal-to-noise ratios [27].

A major issue in the analysis of time series data is that many time-series analysis approaches assume that the observed process is stationary. When the system statistical properties changes over time, the system is non-stationary and consistent comparison of the system status is not possible. Nonstationarity is common in the structures subjected to moving load or ambient excitation as ambient load often produces non-stationary behavior [29, 30]. Successful application of symbolic analysis to study non-stationary systems has been reported in several research [31-33]. Symbolic descriptions of the dynamics of non-stationary Electroencephalography (EEG) signals has been employed to detect changes associated with the stimuli [31]. In another study, symbolic time series analysis has been used to evaluate heart-rate dynamics using heartbeat time series which is highly non-stationary process [32]. Symbolic dynamics and the set of statistical measures built upon it represent a solid framework to address the main challenges of the analysis of non-stationary time data.

Based on the above discussion, symbolic dynamics is a methodology with low computational cost, high robustness against certain level of noise and capable of evaluating non-stationary dynamical systems. These characteristics are highly demanded for implementation in real-time observation application such as structural health monitoring.

Assume a real-valued time series data $X=\left[\left\{x_{1}\right\},\left\{x_{2}\right\}, \ldots,\left\{x_{T}\right\}\right]_{N \times T}$ obtained from a set of observations of an unknown dynamical system. $X$ represents the evolution of the system's dynamics over time; $\left\{x_{t}\right\}_{N \times 1}, t \in[1: T]$ is dated observed vector with length $N$ describing the statues of the system at stage or date $t$ of these measurements, $\left\{x_{t}\right\}_{N \times 1}=\{x(\tau), x(\tau+\Delta), x(\tau+2 \Delta), \ldots, x(\tau+N \Delta)\}^{T} ; \Delta$ is the time delay and $\tau$ refers to the starting point; $N$ is the dimension of the measurement.

Let $\Omega$ be the state space of this varying dynamical system that consists of all possible values of the observed time series. In order to obtain a symbolic representation of $X$, the state space of the underlying system is partitioned into a finite number of subsets $\lambda_{1}, \lambda_{2}, \ldots, \lambda_{S}$ that satisfies the followings, 


$$
\begin{gathered}
\bigcup_{i=1}^{S} \lambda_{i}=\Lambda \\
\lambda_{j} \cap \lambda_{i}=0, i \neq j
\end{gathered}
$$

where $\lambda$ refers to each subset in symbol space; $\Lambda$ represents the whole space and $S$ is the number of partitions. Based on Eq. 1, there is no overlap between two adjacent subsets and they are mutually exclusive. In addition, the subsets are exhaustive and they cover the entire of the state space. Each subset in the state space is assigned to a symbol alphabet that labels that region. Having constructed the symbol space, each measurement point of $\left\{x_{t}\right\}$ is transformed into a symbol by mapping from state space to symbol space $\Psi: \Omega \rightarrow \Lambda$. Therefore the sequence of data in $\left\{x_{t}\right\}$ is described by string of $N$ symbols including $\lambda_{i}, i \in[1: S], S<<N$. The corresponding symbols are the simplified abstraction of the original complex systems.

As an example, suppose a time series $\left\{x_{t}\right\}_{40 \times 1}$ with dimension $N=40$ which varies between -4 and 8 ; A symbol space can be created in a way that uniformly is partitioned into three symbols, $S=3$ as follows, (Fig.1):

$$
\begin{aligned}
& -4<\lambda_{1} \leq 0 \\
& 0<\lambda_{2} \leq 4 \\
& 4<\lambda_{3} \leq 8
\end{aligned}
$$

According to this symbol space, $\left\{x_{t}\right\}_{1 \times 40}$ is uniquely mapped to the following symbol sequence with length 40, [ACBBAABAABBBBAABABBBBBCAABBACBBAAAAABABB].

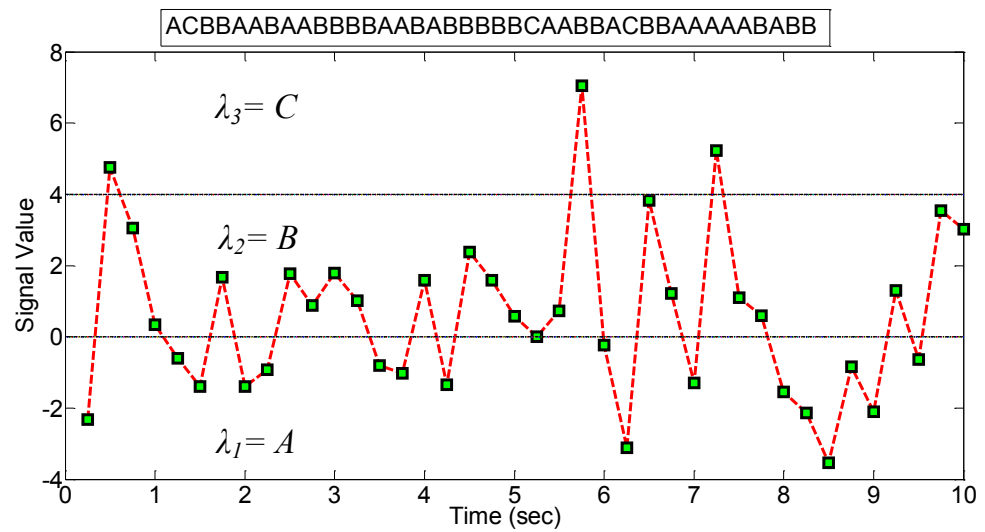

Figure 1. Illustration of mapping from state space to symbol space

It is apparent that the way of discretization of the state space as well as the number of alphabet size have crucial impact on characteristics of the coded or symbolic representation. The choice of alphabet size depends on specific problem, noise level and also the available computation power. A large alphabet may be noise-sensitive while a small alphabet could miss the details of signal dynamics. One way to statistically characterize the obtained symbol sequence is to use Shannon entropy which was first introduced by Claude E. Shannon in 1948 [34]. Shannon entropy is a measure of the complexity and uncertainty of the system under study. The more orderly the system is, the lower will be the entropy, and vice versa. Shannon entropy for a given symbol sequence can be approximated according to Eq. 3, by calculating the probability of occurrence of each symbol, $p\left(\lambda_{i}\right) . p\left(\lambda_{i}\right)$ can be obtained by counting the number of each symbol $\lambda_{i}, i \in[1: S]$ that is found in the sequence divided by the length of the sequence.

$$
H(S)=-\sum_{i=1}^{i=S} p\left(\lambda_{i}\right) \log _{2} p\left(\lambda_{i}\right)
$$


where $H(1)=0$, since in the case of just one partition $(S=1)$ the probability of occurrence of that single symbol will be one.

And in the case of $p_{i}=0$,

$$
\begin{aligned}
& \lim _{p_{i} \rightarrow 0} p\left(\lambda_{i}\right) \log p\left(\lambda_{i}\right)=0 \\
& \text { a }
\end{aligned}
$$

Zero probability for a symbol, $p\left(\lambda_{i}\right)=0$ shows that particular symbol is not observed in the symbol sequence. On the other hand, once the symbols are equally distributed in a sequence $\left(p\left(\lambda_{i}\right)=p\left(\lambda_{j}\right), i \neq j\right)$ maximum entropy is obtained.

In the symbol sequence obtained in Fig.1, the probability of occurrence of symbols are respectively, $p\left(\lambda_{1}\right)=17 / 40, p\left(\lambda_{2}\right)=20 / 40$ and $p\left(\lambda_{3}\right)=3 / 40$.

It can be seen that Shannon entropy is maximized when probabilities are equally distributed and it is minimized once all the probability is concentrated in just one component and other components do not exist in the observation. Higher Shannon entropy indicates there is no way to predict the outcome of the symbol sequences which is refereed to more randomness.

As seen in Eq. 3, Shannon entropy is a function of alphabet size, $S$. Higher number of partitions, $S$, corresponds to retaining the most detailed information of the original measurement such as noise which leads to redundancy and more computational effort. On the contrary, lower number of subsets might lead to loss of information embedded in time series data.

A key issue in partitioning the state space is to make a proper decision on alphabet size, $S$. This issue can be resolved based on the concept of dynamic entropy [35]. Dynamic entropy can be approximated by the Shannon entropy excess associated with addition of one symbol size, $S \rightarrow S+1$ as follows,

$$
h(S)=H(S+1)-H(S)
$$

Large number of partitions $(S \rightarrow N)$ produces smaller dynamic entropy and results in more computational effort on subsequent analysis since there is no considerable reduction in dimension of the original system. On the other hand, small alphabet size corresponds to large dynamic entropy which might not be appropriate due to loss of information. Therefore, a proper selection of number of partitions is a trade-off between efficiency and accuracy.

\section{Damage Localization Based on Symbolic Time Series Approach}

This section presents application of STSA in damage localization which includes four major steps explained in the following parts.

Suppose a gradually time varying system is under observation at $T+1$ dates or stages as $X^{j}=\left[\left\{x_{0}^{j}\right\},\left\{x_{1}^{j}\right\},\left\{x_{2}^{j}\right\}, \ldots,\left\{x_{T}^{j}\right\}\right]_{N \times(T+1)} . X^{j}$ contains all measured signals in this period of observation obtained from $j$-th sensor. $\left\{x_{t}^{j}\right\}$ is a vector of $N$ elements describing the state of the structure in $j$-th sensor, $j \in[1: M]$ at date $t, t \in[0: T] .\left\{x_{0}^{j}\right\}_{N \times 1}$ represents the nominal state of the system in $j$-th sensor location which can be referred to the first set of observations in the system under monitoring. It should be noted that the nominal state is not necessarily the healthy state of the structure.

As an example, if we monitor the integrity of a structure by using 20 sensors over a month by daily observations (31 days and one observation per day); therefore, parameters $M$ (number of sensors) 
and $T$ (number of observations) are, respectively, 20 and 31. $\left\{x_{t}^{j}\right\}$ is the observed response in one of the sensors ( $[j \in 1: 20])$ during this period $t \in[1: 31]$. The observed signal has a length of $N$ elements, let's say starting observation at 10:00 AM until 10:05 AM with time delay 0.01 sec, therefore, $\mathrm{N}=30,000$.

Totally, there is an array of $\mathrm{M}$ sensors recording the response of the structure over time. In order to analyze this huge amount of data, $T$ (dates) $\times M$ (sensors) $\times N$ (length of the observed signal in each sensor), STSA has been conducted to reduce the dimension of information and extract useful features suitable for damage localization paradigm.

\subsection{Reconstruct the time series data by employing discrete wavelet transform (DWT)}

In "real-life" applications and field test experiments, measured data are always susceptible to contamination by measurement noise. Noise can significantly affect the damage characterization results in structural health monitoring $(S H M)$ applications and leads to unreliable and false prediction of structural damage. Hence, to achieve more consistent damage characterization results it is necessary to improve signal to noise ratio $(S N R)$ of measured signals. Application of wavelet transform in noise mitigation has been repeatedly demonstrated in the literature [36-40].

In DWT, filters of different cut-off frequencies are used to analyses the signal. The procedure starts with level one decomposition by passing a half-band low-pass and high-pass filters through the signal. The decomposition process can be mathematically described by convolution operation as

$$
\begin{aligned}
& y_{\text {high }}[k]=\sum_{n} x[n] g[2 k-n] \\
& y_{\text {low }}[k]=\sum_{n} x[n] h[2 k-n]
\end{aligned}
$$

Where $g$ and $h$ are, respectively, associated with high-pass and low-pass filters.

The output of low-pass filter is then passed through the same low-pass and high-pass filters for further decompositions. The decomposition process can be iterated, with successive approximations (low frequency components) being decomposed in turn.

If decomposition is continued to level $J$, then the DWT of the original signal is obtained by concatenating all the coefficients starting from the last level of decomposition as,

$$
x=A^{J}+\sum_{j=-\infty}^{J} D^{j}
$$

Where $A^{j}$ and $D^{j}$, respectively, represent the approximation and detail coefficients at level $j$. DWT at level $J$, filters the signal to low and high frequency portions with the bandwidth dictated by $J$.

Having conducted wavelet decomposition, the denoising operation can be performed by recovering the detail wavelet coefficients. Therefore, detail coefficients with small magnitude can be considered as pure noise and be set to zero. In this regard, each coefficient is compared with a predefined threshold value to decide whether it constitutes a desirable part of the signal or not. As a result, appropriate selection of the threshold value is a crucial decision in this technique. If the threshold is too small or too large, then the procedure tends to over-fit or to under-fit the data. In this study soft threshold approach was utilized as follows,

$$
\delta= \begin{cases}0 & \text { if }|\mathrm{d}| \leq \zeta \\ d-\zeta & \text { if } \mathrm{d}>\zeta \\ d+\zeta & \text { if } \mathrm{d}<-\zeta\end{cases}
$$

where $\zeta, d, \delta$ are, respectively, threshold value, original detail coefficient and recovered (modified) detail coefficient. In this study, threshold value was determined based on universal threshold approach [41] as, 


$$
\zeta \propto \sqrt{2 \log N}
$$

where $N$ is the number of data in the signal as explained earlier.

The denoising procedure ends with reconstructing the signal from sub-bands with smaller bandwidths by taking inverse discrete wavelet transform (IDWT). In this step, the recovered signal is constructed based on the original approximation coefficients of level $J$ and the modified detail coefficients of levels from 1 to $J$.

In this study, time series data have been denoised based on the procedure explained in this section to reduce unwanted noisy part of the signal and produce more consistent damage localization results.

\subsection{Partitioning the state space based on maximum entropy}

In the previous section, recovered version of time series data was generated, $\left\{y_{t}^{j}\right\}_{N \times 1}$; where $\left\{y_{t}^{j}\right\}_{N \times 1}$ denotes recovered version of the observed signal $\left\{x_{t}^{j}\right\}_{N \times 1}$ in $j$-th sensor location, $j \in[1: M]$ at date $t$, $t \in[0: T]$. Then, the normalized signal $\left\{\widetilde{y}_{t}^{j}\right\}_{N \times 1}$ can be easily obtained as follows:

$$
\left\{\tilde{y}_{t}^{j}\right\}=\frac{\left\{y_{t}^{j}\right\}-\mu_{t}^{j}}{\sigma_{t}^{j}}
$$

where $\mu_{t}^{j}$ and $\sigma_{t}^{j}$ are respectively, the mean value and standard deviation of $\left\{y_{t}^{j}\right\}$. This transformation constitutes a unit free measure and makes the data more comparable which is beneficial in damage localization procedure.

Therefore, $Y^{j}=\left[\left\{\tilde{y}_{0}^{j}\right\},\left\{\tilde{y}_{1}^{j}\right\},\left\{\tilde{y}_{2}^{j}\right\}, \ldots,\left\{\tilde{y}_{T}^{j}\right\}\right]_{N \times(T+1)}$ will be employed in the subsequent analysis.

The damage localization procedure is followed by generating symbol sequences of data. As mentioned earlier in section 2, in order to generate the symbol sequences, first the symbol space should be created. Totally, there are $M$ sensor locations and therefore, $M$ distinct symbol spaces will be generated.

In this regard, two main decisions should be made: first, the alphabet size and second, the way of partitioning for each symbol space. These decisions are made based on the nominal state of the $j$-th sensor location, $\left\{\tilde{y}_{0}^{j}\right\}$ and after creating the symbol space for that particular location, it remains invariant as a basis to generate the symbol sequences of the following observations $t \in[1: T]$ for that particular location. It is clear that for two different sensor locations, $i \neq j$ different symbol spaces are acquired according to their nominal states, $\left\{\widetilde{y}_{0}^{i}\right\}$ and $\left\{\widetilde{y}_{0}^{j}\right\}$. It is worth mentioning that the nominal state is not necessarily the healthy state of the structure and it only refers to the state of the structure at the first stage of monitoring.

In this study, partitioning of symbol space is performed based on maximum entropy approach. In this scheme, sparsity and distribution of data is considered as a key factor to generate the partitions. It means that highly-dense regions in terms of information are partitioned finer and sparse regions are devoted fewer partitions; to illustrate this fact, an example is examined.

Suppose for arbitrary sensor location $j$, the partitioning of symbol space is created based on $\left\{\widetilde{y}_{0}^{j}\right\}$ with $S_{j}$ partitions, as $\lambda_{1}, \lambda_{2}, \ldots$ and $\lambda_{S_{j}} \cdot\left\{\tilde{y}_{0}^{j}\right\}$ is a vector with length $N$ whose elements vary between $\left.\left\{\tilde{y}_{0}^{j}\right\}\right|_{\text {min }}$ and $\left.\left\{\widetilde{y}_{0}^{j}\right\}\right|_{\max }$. 
Partitioning of the symbol space can be carried out based on either uniform entropy approach or maximum entropy approach. In the first approach, the space is divided to equal-sized partitions as,

$$
\begin{aligned}
& \left.\left\{\tilde{y}_{0}^{j}\right\}\right|_{\text {min }} \leq \lambda_{1} \leq\left.\left\{\tilde{y}_{0}^{j}\right\}\right|_{\text {min }}+\left(\left.\left\{\tilde{y}_{0}^{j}\right\}\right|_{\text {max }}-\left.\left\{\tilde{y}_{0}^{j}\right\}\right|_{\text {min }}\right) / S_{j} \\
& \left.\left\{\widetilde{y}_{0}^{j}\right\}\right|_{\text {min }}+\left(\left.\left\{\tilde{y}_{0}^{j}\right\}\right|_{\text {max }}-\left.\left\{\widetilde{y}_{0}^{j}\right\}\right|_{\text {min }}\right) / S_{j} \leq \lambda_{2} \leq\left.\left\{\widetilde{y}_{0}^{j}\right\}\right|_{\text {min }}+2\left(\left.\left\{\widetilde{y}_{0}^{j}\right\}\right|_{\text {max }}-\left.\left\{\widetilde{y}_{0}^{j}\right\}\right|_{\text {min }}\right) / S_{j} \\
& , \ldots \\
& \left.\left\{\tilde{y}_{0}^{j}\right\}\right|_{\text {min }}+\left(S_{j}-1\right)\left(\left.\left\{\tilde{y}_{0}^{j}\right\}\right|_{\text {max }}-\left.\left\{\tilde{y}_{0}^{j}\right\}\right|_{\text {min }}\right) / S_{j} \leq \lambda_{S_{j}} \leq\left.\left\{\tilde{y}_{0}^{j}\right\}\right|_{\text {max }}
\end{aligned}
$$

while in the second approach, partitions are not equal-sized. In the maximum entropy approach, first, the elements of $\left\{\tilde{y}_{0}^{j}\right\}$ are ordered from lesser to greater; therefore, the sorted vector, $\left\{\overline{\tilde{y}}_{0}^{j}\right\}$ starts with $\left.\left\{\tilde{y}_{0}^{j}\right\}\right|_{\min }$ and ends with $\left.\left\{\tilde{y}_{0}^{j}\right\}\right|_{\max }$. Then the arranged vector is divided to $N / S_{j}$ sections, each of them makes one partition of the space as follows,

$$
\begin{aligned}
& \left.\left\{\widetilde{y}_{0}^{j}\right\}\right|_{\min } \leq \lambda_{1} \leq \overline{\widetilde{y}}_{0}^{j}\left[N / S_{j}\right] \\
& \overline{\widetilde{y}}_{0}^{j}\left[N / S_{j}\right] \leq \lambda_{2} \leq \overline{\widetilde{y}}_{0}^{j}\left[2 N / S_{j}\right] \\
& , \ldots \\
& \overline{\tilde{y}}_{0}^{j}\left[\left(S_{j}-1\right) N / S_{j}\right] \leq \lambda_{S_{j}} \leq\left.\left\{\widetilde{y}_{0}^{j}\right\}\right|_{\max }
\end{aligned}
$$

where $\overline{\widetilde{y}}_{0}^{j}\left[N / S_{j}\right]$ denotes the $\left(N / S_{j}\right)-$ th element in vector $\left\{\overline{\widetilde{y}}_{0}^{j}\right\}$.

Fig. 2 compares the symbol space for $j$-th sensor location, $\Lambda_{j}$ obtained by these two approaches; Fig.2 (a) and Fig.2 (b) respectively, illustrate symbol space based on uniform entropy and maximum entropy. As expected, the second approach, maximum entropy, devotes more partitions to information-rich areas, unlike the first technique in which the partitions are equal-sized. In the first approach, we see that the probability of symbols is not the same, as the number of data in each partition is different with one another; while the symbol sequence generated by the second approach provides equally distributed probabilities at nominal state. This is interestingly attractive since by comparing the symbol probabilities of future observations $t \in[1: T]$ with the nominal state, it is possible to monitor any variation in the system. Therefore, the symbol sequence generated based on this symbol space will be better indicator of any evolving damage in the structure and will be used in this study.

(a)

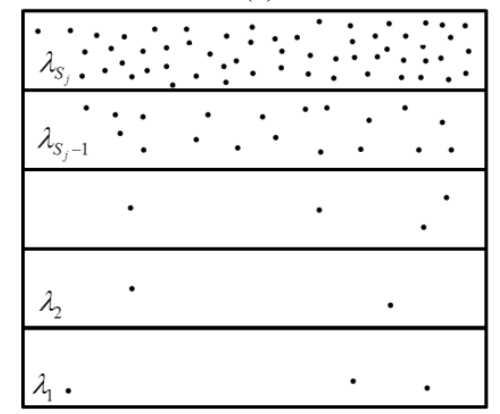

$\Lambda_{j}$ (b)

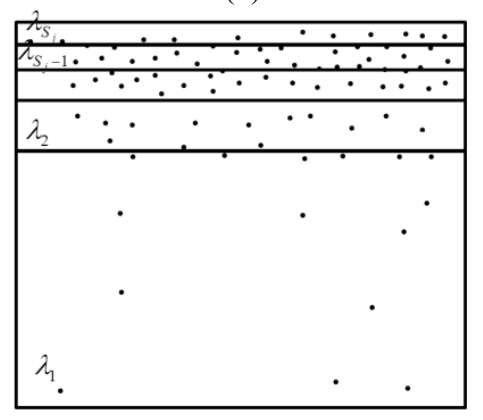

$\Lambda_{j}$

Figure 2. Space partitioning based on, (a) uniform entropy, (b) maximum entropy. 


\subsection{Selection of alphabet size}

The size of alphabet for $j$-th sensor location, $S_{j}$ is another issue to be determined. In this study, the same alphabet size is considered for all sensor locations, $S_{i}=S_{j}=S, i \neq j$. This is because, in the monitoring process, the state of each location is compared with other locations in a statistical sense by comparing anomaly measures of different locations. In order to compare the statistical behavior of two distinct locations, it is evident that the probabilities should be of the same length to make this comparison possible.

The selection of alphabet size can be done in a straightforward way based on dynamic entropy of symbol sequence in the nominal state and prescribed threshold value according to Eq. 5. As mentioned earlier, in this work, partitioning is done based on maximum entropy approach; therefore, in the nominal state, the probabilities of symbols for two different symbols $\lambda_{m}$ and $\lambda_{n}$ are the same $p\left(\lambda_{m}\right)=p\left(\lambda_{n}\right), m \neq n$, therefore Eq. 3 can be rewritten as

$$
H(S)=-\sum_{i=1}^{i=S} \frac{1}{S} \log _{2} \frac{1}{S}
$$

The procedure of selecting alphabet size starts with $S=2$ and then Shannon entropy excess is calculated with the addition of one symbol size $S=2+1$ as follows,

$$
\begin{aligned}
& S=2 \Rightarrow H(2)=-\left(\frac{1}{2} \log \frac{1}{2}+\frac{1}{2} \log \frac{1}{2}\right)=0.6931 \\
& \mathrm{~S}=3 \Rightarrow H(3)=-\left(\frac{1}{3} \log \frac{1}{3}+\frac{1}{3} \log \frac{1}{3}+\frac{1}{3} \log \frac{1}{3}\right)=1.0986 \Rightarrow \mathrm{h}(2)=H(3)-H(2)=0.4055 \\
& \mathrm{~S}=4 \Rightarrow H(4)=-\left(\frac{1}{4} \log \frac{1}{4}+\frac{1}{4} \log \frac{1}{4}+\frac{1}{4} \log \frac{1}{4}\right)=1.3863 \Rightarrow \mathrm{h}(3)=H(4)-H(3)=0.2877 \\
& \mathrm{~S}=5 \Rightarrow H(5)=-\left(\frac{1}{5} \log \frac{1}{5}+\frac{1}{5} \log \frac{1}{5}+\frac{1}{5} \log \frac{1}{5}\right)=1.6094 \Rightarrow \mathrm{h}(4)=H(5)-H(4)=0.2231
\end{aligned}
$$

As illustrated by Eq. 14, by increasing $S: 2 \rightarrow 3$, the Shannon entropy excess will be 0.4055 . If the procedure continues for $S=3 \rightarrow 4$, a less dynamic entropy is obtained, 0.2877 . Therefore, by increasing alphabet size dynamic entropy reduces to zero as $\mathrm{S} \rightarrow \mathrm{N} \Rightarrow \mathrm{h} \rightarrow 0$. This operation keeps until dynamic entropy is less than a predefined threshold value.

\subsection{Developing the anomaly measures for damage localization}

The procedure of damage localization so far consisted of reconstructing the noisy data of time series signals and generating the symbol space for each sensor location based on its nominal state. According to these foundations, at each stage of measurement $t \in[1: T]$ and for each sensor location $j \in[1: M]$, the symbol sequences are generated. Therefore, there will be $T \times M$ symbol sequences each with length of $N$.

For each symbol sequence, the probability of symbols, $p_{t}^{j}\left(\lambda_{k}\right)$, is found by the number of occurrence of that specific symbol divided by the length of the sequence, $N . p_{t}^{j}\left(\lambda_{k}\right), k \in[1: S]$ refers to the number of occurrence of symbol $\lambda_{k}$ in the symbol sequence obtained for $j$-th sensor at stage $t$. On this point, probability vector $\mathbf{P}_{\mathbf{t}}^{\mathbf{j}}$ is defined as a vector containing the probabilities of all symbols in $j$-th sensor at date $t$ as follows, 


$$
\mathbf{P}_{\mathbf{t}}^{\mathbf{j}}=\left[p_{t}^{j}\left(\lambda_{1}\right), p_{t}^{j}\left(\lambda_{2}\right), \ldots, p_{t}^{j}\left(\lambda_{S}\right)\right]
$$

As expected the probability vector for any arbitrary location at $t=0$, nominal state, will be,

$$
\mathbf{P}_{\mathbf{0}}^{\mathbf{j}}=\left[\frac{1}{S}, \frac{1}{S}, \ldots, \frac{1}{S}\right]
$$

Comparing probability vectors at time $t, \mathbf{P}_{\mathbf{t}}^{\mathbf{j}}$ with its nominal state, $\mathbf{P}_{\mathbf{0}}^{\mathbf{j}}$ leads to assess any possible change in the system behavior in $j$-th location. To make this comparison feasible, anomaly measure is defined as,

$$
A_{t}^{j}=A\left(\mathbf{P}_{\mathbf{t}}^{\mathbf{j}}, \mathbf{P}_{\mathbf{0}}^{\mathbf{j}}\right)=\left\|\mathbf{P}_{\mathbf{t}}^{\mathbf{j}}\right\| \mathbf{P}_{\mathbf{0}}^{\mathbf{j}} \|
$$

Anomaly measure is a scalar value representing the farness of the system from its nominal state. The more the anomaly measure is, the less closeness is between current state and the nominal state of the system; which is an indicator of damage presence in the structure.

The last step of damage localization is to compare the anomaly measures of different locations to detect any sudden variation which corresponds to damage. Let's say at date $t$ the anomaly measures for $M$ sensor locations are obtained and compared together. At damage location, it is expected to get abrupt change in anomaly measure since the statistical behavior of this location is the most deviated from its nominal state amongst all other locations. This idea gives a hint that if the anomaly measures are developed in different locations, at damage location, higher anomaly is obtained; therefore, damage localization index at stage $t$ is defined as:

$$
\Delta_{t}=\max A_{t}^{j} \quad, j=1: M
$$

$\Delta_{t}$ refers to the location of maximum anomaly measure amongst all measured points at stage $t$ which corresponds to the location of damage.

In summary, the presented damage localization technique includes the following steps:

1. Denoise time series data by employing DWT.

2. Generate the symbol space for each sensor location according to the nominal state.

3. Generate the symbol sequences, probability vectors and anomaly measures.

4. Localize damage by comparing the anomaly measures of different measured points.

As will be illustrated, the proposed damage localization method is robust in presence of noise influences which leads to more consistent damage localization results in real-life applications.

\section{Application}

The damage localization test derived in this paper is applied on two numerical examples to evaluate the feasibility and effectiveness of the method; (1) a flexural beam and (2) a 2-D planar truss structure. Damage localization scheme was performed considering different damage conditions in presence of practical uncertainties such as noise and incompleteness of measured data.

\subsection{A Flexural Beam}

In the first demonstration, a rectangular steel beam with dimensions $2000 \mathrm{~mm} \times 40 \mathrm{~mm} \times 40 \mathrm{~mm}$ and material properties $E=200 G P a, \rho=7800 \mathrm{~kg} / \mathrm{m}^{3}$ and Poisson ratio $=0.3$ is considered. Since the aspect ratio of the beam is $1 / 50$, hence, the behavior of the beam can be modeled, with reasonable 
accuracy, by Euler-Bernoulli beam theory. The FE model of the structure was developed with the help of an in-house finite element (FE) code implemented in Matlab. The beam is discretized by 20 linear beam finite elements with two lateral and rotational degrees of freedom at each node. Two different boundary conditions, fixed-free (C-F) and two-fixed-end (C-C) supports, are considered as shown, respectively, in Fig.3 (a) and Fig.3 (b). Rayleigh damping was adopted for constructing the damping matrix.

(a)

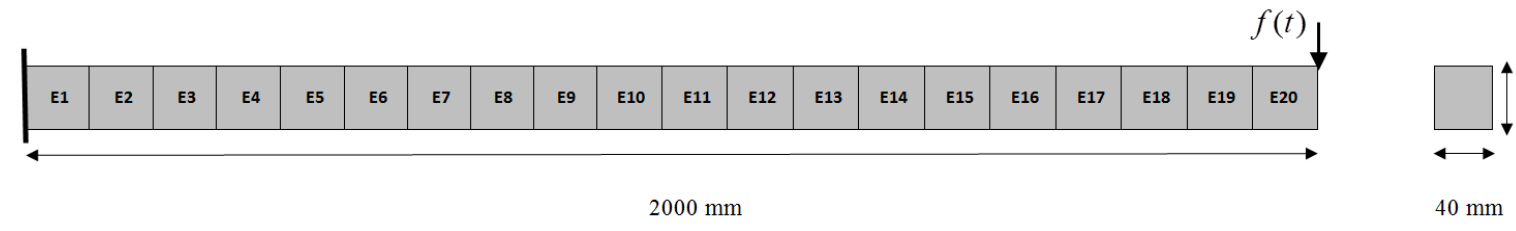

(b)

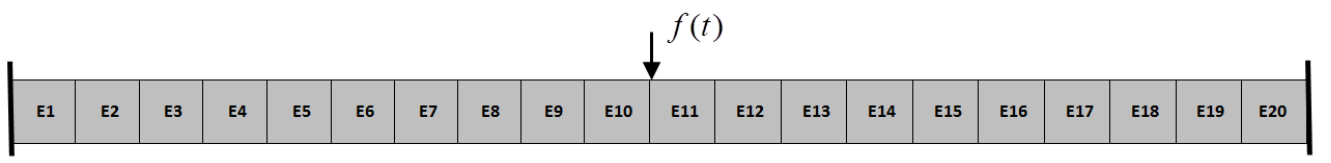

Figure 3. Illustration of the flexural beam with different boundary conditions, (a) fixed-free beam (C-F), (b) two-fixed-end beam (C-C).

The beam is assumed to be a time-variant mechanical system subjected to gradual stiffness degradation at particular element locations which is simulated by introducing Elastic modulus reduction. It is commonly accepted that for the vast majority of the structures, stiffness decay due to damage evolution process can be modelled by a scheme shown in Fig. 4 (a), [42-45] . As exhibited, in the initial stages, deterioration occures in an approximately linear and slight fashion follwed by abrupt and rapid reduction in the stiffness which consequently results in failure. Based on this figure, $E_{d}$ (Elastic modulus of the damaged element) starts with 200GPa which corresponds to the nominal state, in this situation is also equivalent with the healthy state, and over 30 stages, it approaches to zero. Fig. 4 (b) indicates the corresponding change in the Elastic modulus at each stage compared to the nominal stage. As expected, an increasing trend is obtained, starting with $0 \%$ change in the Elastic modulus at stage 1 and ending with 100\% change in the Elastic modulus at stage 30.
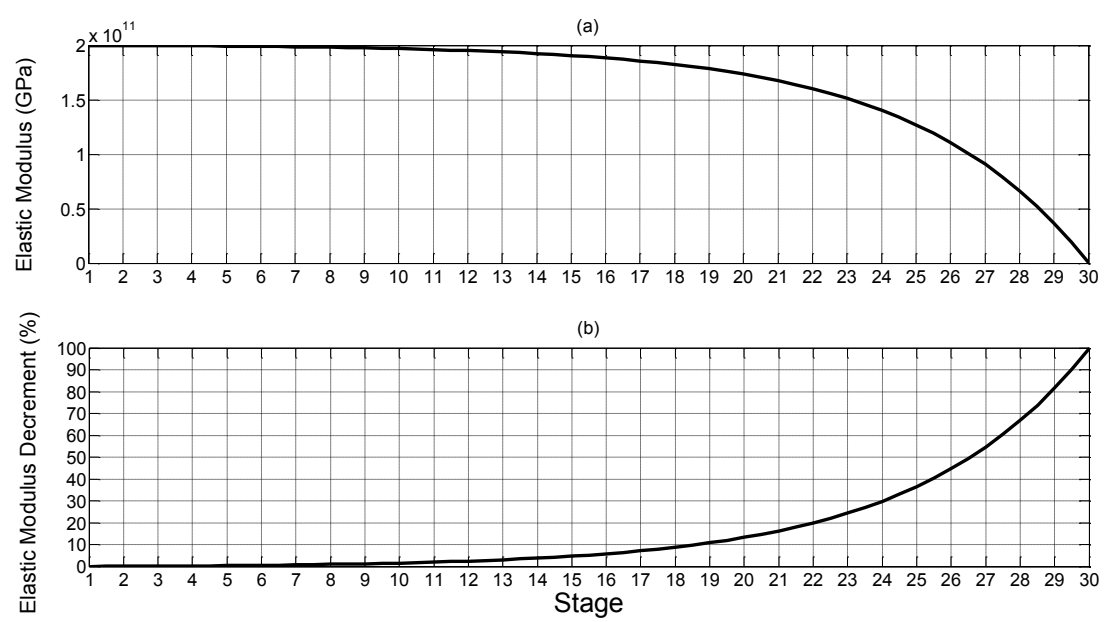

Figure 4. Deterioration history, (a) Elastic Modulus of the damaged element, (b) The change in the Elastic Modulus compared to the nominal state $(\%)$. 


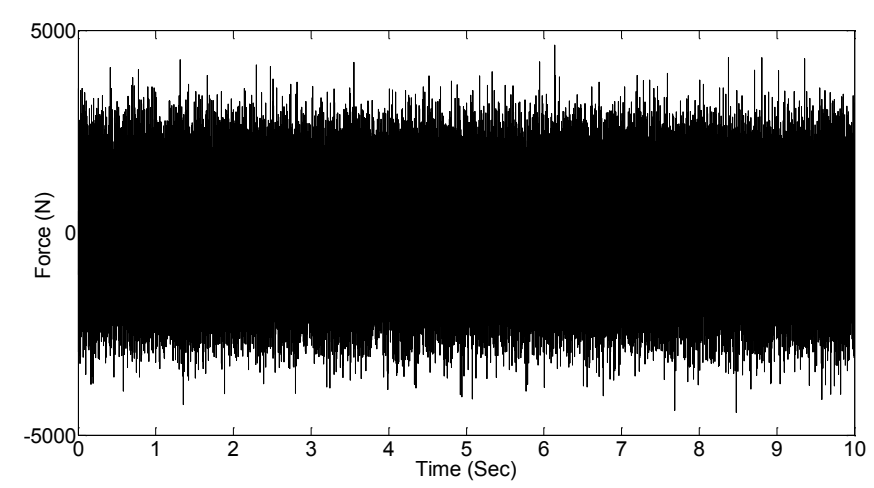

Figure 5. Gaussian excitation

The structure is subjected to Gaussian stationary white noise excitation as shown in Fig. 5. The location of input excitation is assumed to be at free end for the C-F case study and at mid-span for the C-C case study.

The time series responses were extracted at each nodal point by introducing state-space vector. At each stage of monitoring, $t \in[0: 30]$, first, the equation of motion is updated based on the current value of $E_{d}$ and then is solved by employing discretization technique to obtain time histories of all discretized points during 10 seconds with time step of 0.1 millisecond. To evaluate the robustness of the method to the changes in the excitation, the input Gaussian excitation is not kept constant and a new random excitation is generated at each stage of monitoring, $t \in[0: 30]$; it is worth mentioning that only the distribution (normal) and the amplitude of the random excitation are kept unchanged.

In order to examine the robustness of the damage localization method in presence of noise influences, the deterministic time responses, $\{\bar{x}\}$, obtained from the previous step, were polluted with $2 \%$ white random noise with zero mean value as follows,

$$
\left\{\hat{x}_{t}^{j}\right\}=\left\{\bar{x}_{t}^{j}\right\}+\left(\frac{e}{100} \cdot \sigma\left(\left\{\bar{x}_{t}^{j}\right\}\right) \cdot R(t)\right)
$$

where $\left\{\bar{x}_{t}^{j}\right\}$ is the deterministic time response in $j$-th sensor at stage $t . \sigma\left(\left\{\bar{x}_{t}^{j}\right\}\right)$ is the standard deviation of the clean response and $R(t)$ generates randomly distributed data with distribution prescribed. $e$ indicates the percentage of noise.

Having obtained the time series responses at all nodal points (20 locations for the C-F beam and 19 locations for the C-C beam), damage localization process starts with reconstructing the time series data by employing DWT. For this implementation, first the mother wavelet (window function), should be selected. Amongst the well-known mother wavelets, Daubechies wavelet is the most commonly used and has gained more popularity due to its useful properties. They are widely used in the field of noise-suppression [46-49]. Daubechies wavelets are a family of orthogonal wavelets and have higher order than any other wavelets. Due to compact support and orthogonally, they are capable of describing the details of the problem conveniently and accurately as well as making discrete wavelet transform practicable. In this work, Daubechies wavelet with order 5 has been employed as a mother to perform DWT up to the fifth level to obtain wavelet coefficients. Matlab command "wden" has been employed to denoise the signals with respect to aforementioned window. Universal threshold approach with soft thresholding has been used to recover the noisy signal. Having denoised the signals, time series data are normalized according to Eq.10 to obtain $\left\{\tilde{y}_{t}^{j}\right\}$. For each measured point, the symbol space was generated based on descriptions in section 3.2 and 3.3. Based on our investigations, it was found that the size of alphabet 4 is appropriate and is considered for all measured locations. After creating the symbol sequences, the probability vectors and anomaly measures were developed according to section 3.4. 


\subsubsection{Damage detection}

The effect of damage on anomaly measure was investigated by using only one sensor. A deterioration was introduced in element 20 in the C-F beam and the anomaly measure was obtained during 30 stages at a sensor located between element 1 and 2, Fig. 6. Element 20 was deliberately selected for this investigation as damage in this element has the lowest impact on the global stiffness of the structure compared to other elements. Also, the choice of measurement point is because it is located in the most distant position from the damage.

As seen in Fig.6, anomaly measure has been plotted only for stages 1 to 24 since after stage 25 the change in the anomaly measure is significant and the early part of the graph, stages 1 to 24 , is almost invisible. Based on Fig.6, an increasing trend in the anomaly measure is obtained after stage 13. Stage 13 corresponds to less than 5\% stiffness reduction in the element (See Fig.4 (b)). As deterioration evolves, higher values of anomaly measure are obtained which means the system is getting far from its nominal state. The more the anomaly measure is, the less closeness is between the current state and the nominal state; which is an indicator of damage presence.

Based on this demonstration, the proposed method can reliably detect gradually evolving deterioration in the structure with small severity $(5 \%)$ by using only one sensor. The other interesting point is that the method is robust to changes in the excitation. As said earlier, at each stage of deterioration, the response of the structure is obtained under a newly generated random excitation.

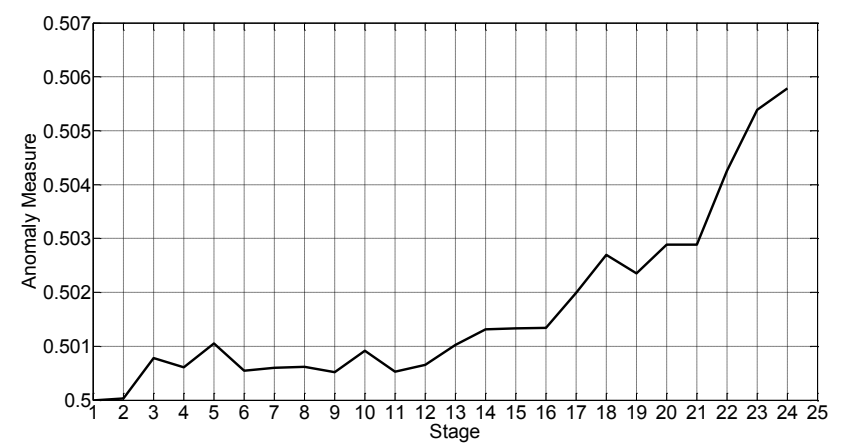

Figure 6 . The impact of deterioration in element 20 on anomaly measure at sensor located between elements 1 and 2

\subsubsection{Damage localization results}

Several damage cases were investigated for $\mathrm{C}-\mathrm{F}$ and $\mathrm{C}-\mathrm{C}$ beams which are tabulated briefly in Table 1: For the C-F beam, six different damage cases are considered: (i) single state damage in element 3 (close to the fixed-end), (ii) single state damage in element 11 (close to mid-span), (iii) single state damage in element 18 (close to the free end), (iv) multiple state damage in elements 3 and 11, (v) multiple state damage in elements 3 and 18, and (vi) multiple state damage in elements 11 and 18 .

For the C-C beam, four different damage cases are considered: (i) single state damage in element 11 (close to mid-span); (ii) single state damage in element 17 (close to the fixed-end); (iii) multiple state damage in elements 11 and 17, (iv) multiple state damage in elements 3 and 17.

Table 1. Introduced damage cases for the flexural beam

\begin{tabular}{|c|c|c|}
\hline Damage cases & C-F beam & $\mathrm{C}-\mathrm{C}$ beam \\
\hline Single state damage & $\begin{array}{l}\text { Element } 3 \\
\text { Element } 11 \\
\text { Element } 18\end{array}$ & $\begin{array}{l}\text { Element } 11 \\
\text { Element } 17\end{array}$ \\
\hline Multiple state damage & $\begin{array}{l}\text { Elements } 3 \text { and } 11 \\
\text { Elements } 3 \text { and } 18 \\
\text { Elements } 11 \text { and } 18\end{array}$ & $\begin{array}{l}\text { Elements } 11 \text { and } 17 \\
\text { Elements } 3 \text { and } 17\end{array}$ \\
\hline
\end{tabular}


The damage localization results are presented in Fig.7 and Fig.8, respectively, for the C-F and C-C beams. These results are based on the observed responses at all nodal points $(20$ sensors for the $\mathrm{C}-\mathrm{F}$ beam and 19 sensors for the C-C beam).

(a)

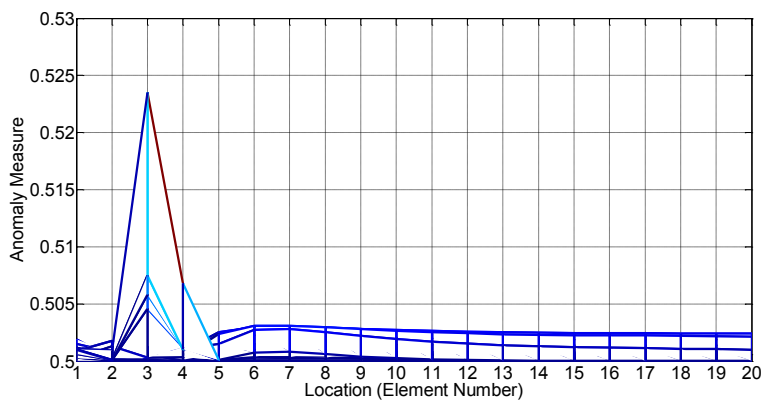

(c)

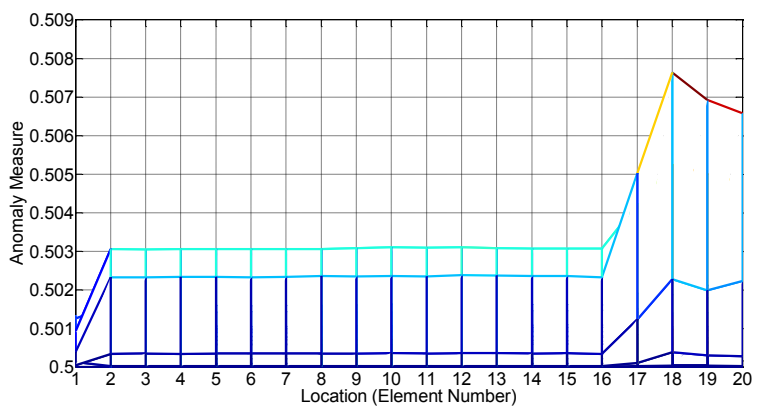

(e)

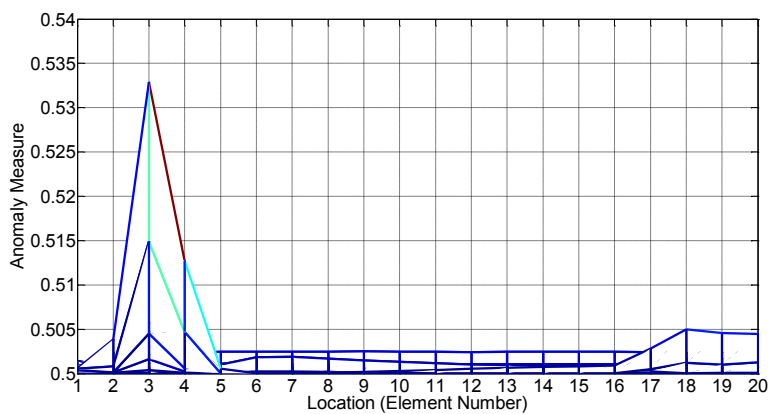

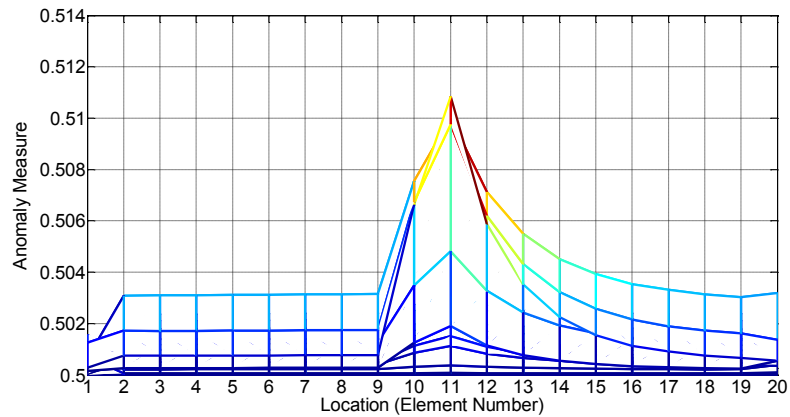

(d)

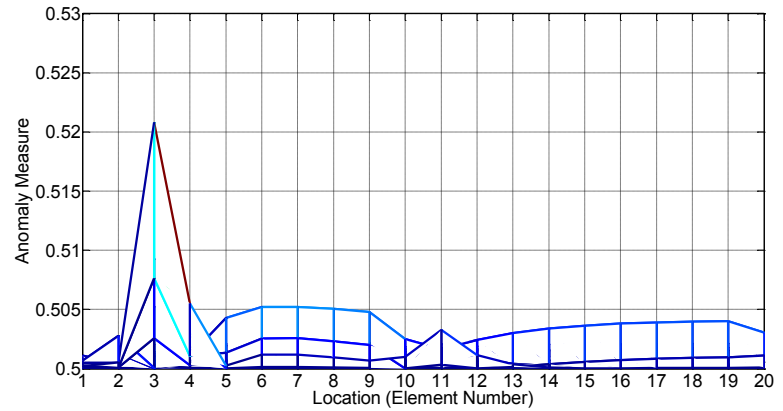

(f)

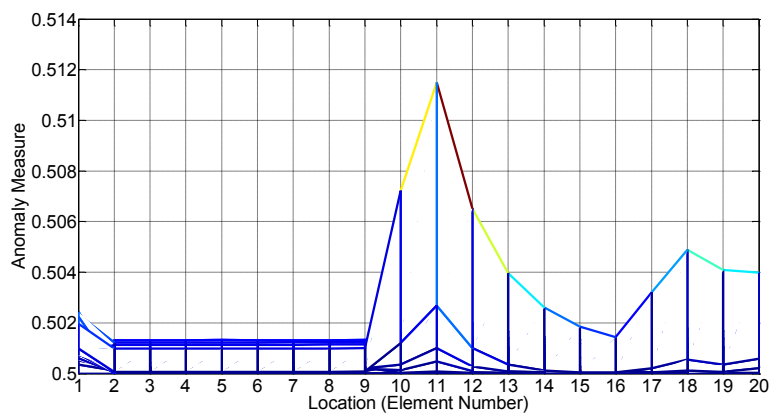

Figure 7. Damage localization results for the C-F beam, (a) single state damage in element 3, (b) single state damage in element 11, (c) single state damage in element 18, (d) multiple state damage in elements 3 and 11 (e) multiple state damage in elements 3 and 18, (f) multiple state damage in elements 11 and 18 .

(a)

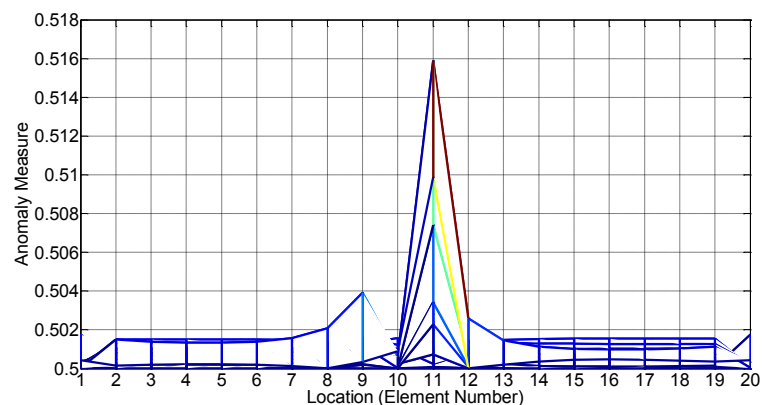

(c) (b)

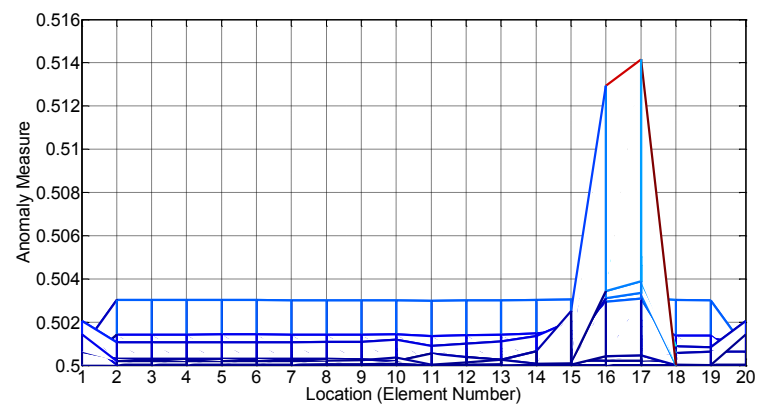

(d) 

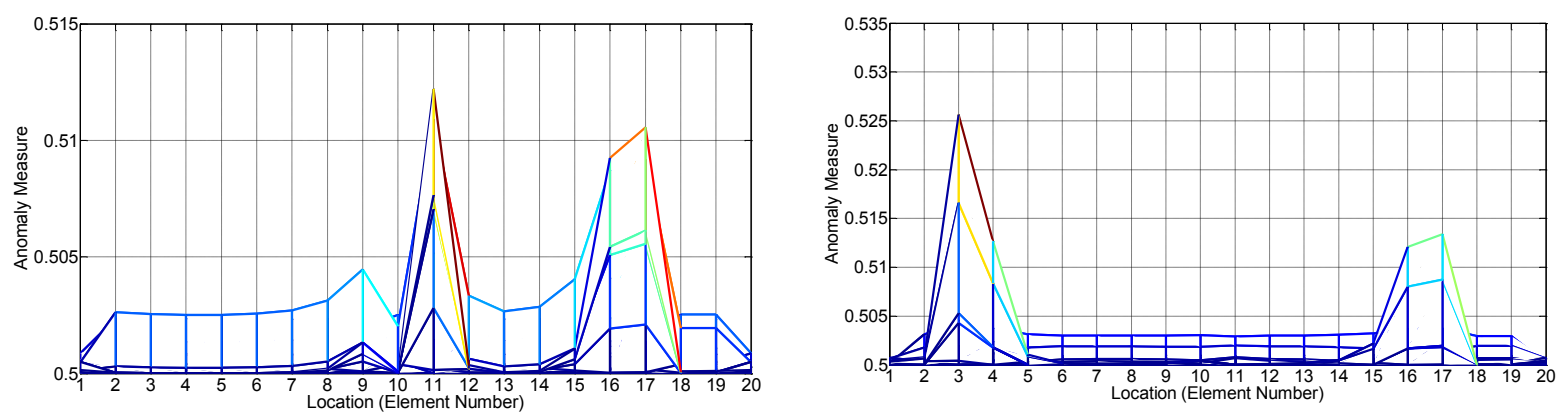

Figure 8. Damage localization results for the C-C beam, (a) single state damage in element 11, (b) single state damage in element 17, (c) multiple state damage in elements 11 and 17, (e) multiple state damage in elements 3 and 17.

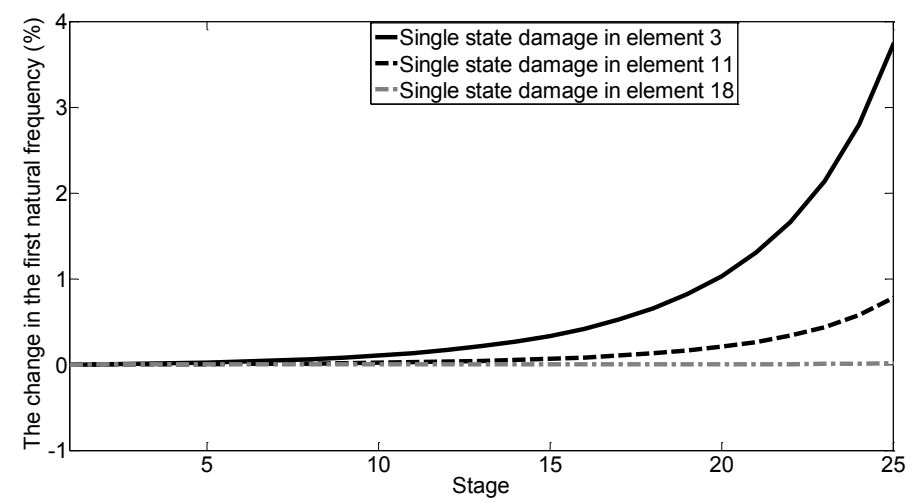

Figure 9. The impact of deterioration on the first natural frequency in the C-F beam.

Before investigating the damage localization results, presented in Fig.7 and Fig. 8, first, the impact of deterioration in different locations on the first natural frequency was investigated for the C-F beam and the result is presented in Fig.9.

Three pre-defined damage cases, (See Table 1), (i) single state damage in element 3, (ii) single state damage in element 11 and (iii) single state damage in element 18 are considered. As shown in Fig.9, as deterioration develops the change in the first natural frequency compared to the baseline state increases. The interesting point lies in the fact that the sensitivity of the change in the first natural frequency increases as damage location moves toward the fixed end since damage close to the fixedend has higher negative impact on the global stiffness of the beam. As depicted, the same severity of the single state damage in elements 3, 11 and 18, respectively, causes $3.72 \%, 0.77 \%$ and $0.01 \%$ changes in the first natural frequency compared to the baseline state at stage 25 . Based on this observation, it is expected that higher damage indices is obtained for damages which have higher adverse effect on the global stiffness of the structure. Therefore, for damages with the same severity, but different locations, we expect that different damage indices are obtained.

With this background, now, let's analyze the damage localization results presented in Fig. 7 and Fig. 8. As can be seen in Fig.7, in the C-F beam, in all damage cases, single or multiple state, the location of damages can be successfully identified without any false prediction. Moreover, by investigating the damage indices obtained for different damage cases in the C-F beam, it can be realized that higher damage indices correspond to more sensitive damage locations. The obtained damage indices for single state damage at elements 3,11 and 18 are respectively 0.5235, 0.5108 and 0.5076 (See Fig.7 (a), Fig.7 (b) and Fig.7 (c)); it confirms our previous statement that the sensitivity of damage index is higher once damage is closer to the fixed-end. The same conclusion can be attained once multiple damage cases are investigated. In all multiple damage cases, the damage that is closer to the fixed end has higher damage index compared to the damage that is closer to the free end. 
In the case of C-C beam, again, the location of all damages can be accurately identified. But, it seems a false alarm appears in element 9 in two damage cases: single state damage in element 11, Fig.8 (a) and multiple state damage in elements 11 and 17, Fig. 8 (c); however, the damage index obtained for element 9 is much smaller compared to the damage indices obtained for real damages.

By investigating Fig.8, it can also be realized that the sensitivity of damage index to the damage close to the mid-span (element 11) is slightly higher than the damage close to the fixed end (element 17); obtained damage indices for single state damage in elements 11 and 17 are respectively, 0.5159 and 0.5141 (See Fig.8 (a) and Fig.8 (b)). The same finding can be obtained from multiple state damage case shown in Fig.8 (c) which attributes higher damage index to the damage close to the mid-span rather damage close to the fixed end.

Base on this investigation, it can be concluded that the proposed method not only can identify the location of damages in single or multiple states but also in the case of damages with the same severity attributes higher damage indices for more sensitive ones.

\subsubsection{Damage localization results using 10 sensors}

Damage localization results presented in the previous section was based on measurement at all nodal points. In this section, the number of sensors is reduced to half and only measurements from 10 nodal points (sensors located at even points: $2,4,6, \ldots, 20$ ) are taken into account.

The damage localization procedure was performed for both cases and the results for the C-F case are presented in Fig.10. According to the presented results in Fig.10, it can be seen that the damage index gets its maximum value at the sensor location which is the closest one to the damage location; for single state damage at elements 3,11 and 18, sensors 4, 12 and 18, respectively, show the maximum damage indices; these sensors are located in the immediate neighborhood of the damaged element, (see Fig.10 (a), Fig.10 (b) and Fig.10 (c)). Based on this observation, it can be concluded that the maximum damage index corresponds to the sensor located in the neighborhood of the damage and with distance from the damage area the damage index decays very quickly. However it should be mentioned that for satisfactory evaluation of damage location it is necessary to distribute sufficient sensors along the structure.

(a)

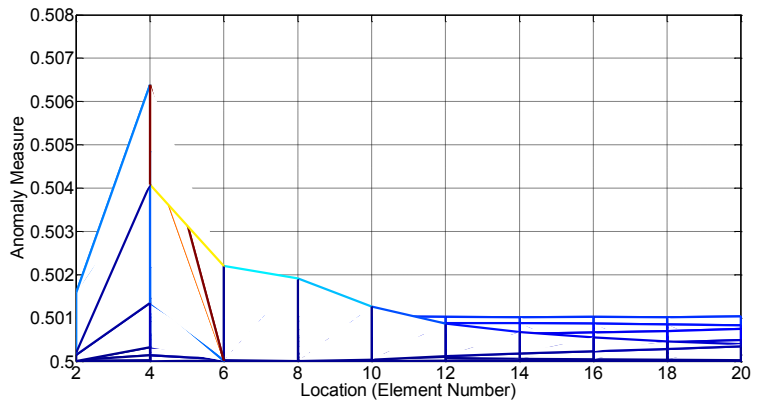

(c)

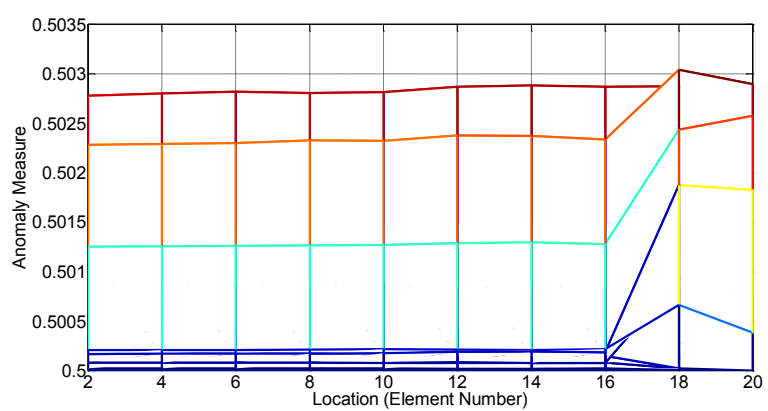

(e) (b)

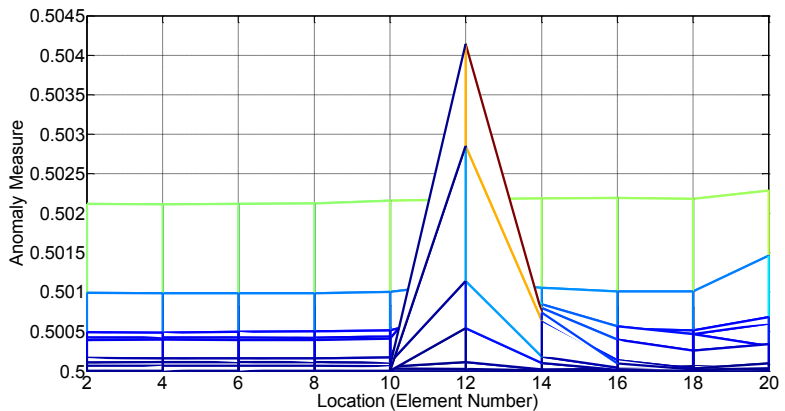

(d)

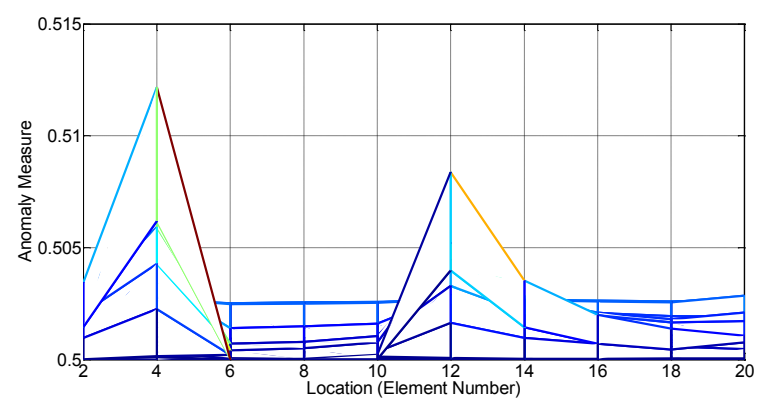

(f) 

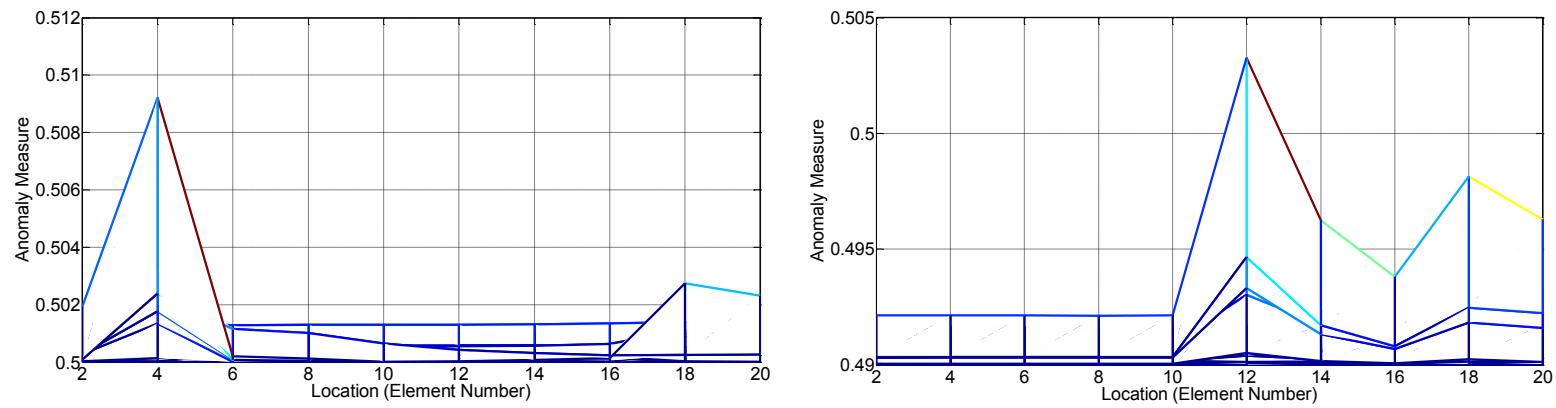

Figure 10. Damage localization results for the C-F beam using 10 sensors, (a) single state damage in element 3, (b) single state damage in element 11, (c) single state damage in element 18, (d) multiple state damage in elements 3 and 11 (e) multiple state damage in elements 3 and 18, (f) multiple state damage in elements 11 and 18 .

\subsection{A Truss Structure}

In the second demonstration, the damage identification strategy is applied on a planar truss bridge to illustrate the applicability of the proposed approach. The truss model has 14 nodal points (underlined numbers), 25 truss elements (bold numbers) and 25 nodal dofs as shown in Fig. 11.

Values for the material and geometric properties are as follows: the elastic modulus $E=200 \mathrm{GPa}$; the cross-sectional area of all elements $=0.01 \mathrm{~m}^{2}$; the length of all horizontal and vertical members $=1 \mathrm{~m}$; the length of the inclined members $=1.41 \mathrm{~m}$; and the mass density $\rho=7800 \mathrm{~kg} / \mathrm{m}^{3}$.

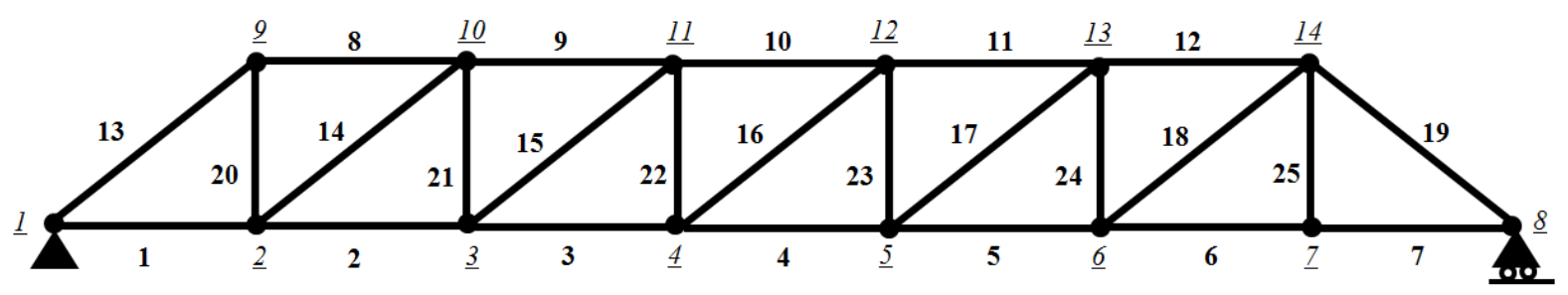

Figure 11. A 2-D planar truss bridge

This type of structure is subjected to vibration caused by the passing traffic or wind; hence, it is reasonable to assume the structure is under ambient excitation to collect structural responses for locating damage. It is supposed that the structure is subjected to random Gaussian excitation synchronously exerted at all generalized coordinates. The response of the structure is recorded both in $x$ and $y$ directions at all nodal points.

A total of 10 damage cases were designed considering damage in horizontal, vertical and diagonal members in single or multiple states (See Table 2). Damage was simulated by gradual decreasing of the member Elastic modulus; the same trend presented in Fig.4 (a) was considered for stiffness deterioration.

At each stage of deterioration, a new random excitation (it should be noted again that the maximum amplitude and the distribution of the excitation are kept constant at all stages) is generated and the time response of the structure is extracted at all dofs by introducing state space vector. At each stage of monitoring, $t \in[0: 30]$, the deterministic time series of strain at element $j,\left\{\bar{\varepsilon}_{t}^{j}\right\}, j \in[1: 25]$ is developed according to the recorded nodal displacements as:

$\left\{\bar{\varepsilon}_{t}^{j}\right\}_{N \times 1}=\frac{1}{L}\left[\left\{\bar{u}_{t}^{a}\right\}\left\{\bar{v}_{t}^{a}(t)\right\}\left\{\bar{u}_{t}^{b}\right\}\left\{\bar{v}_{t}^{b}\right\}\right] \cdot[-\cos (\theta)-\sin (\theta) \cos (\theta) \sin (\theta)]^{T}$

where, $a$ and $b$ are nodal points of member $j ;\left\{\bar{u}_{t}^{a}\right\}_{N \times 1}$ and $\left\{\bar{v}_{t}^{a}\right\}_{N \times 1}$ are respectively, deterministic recorded time responses at node number $a$, at stage $t$, in $x$ and $y$ directions. $L$ and $\theta$ respectively, indicate the length of the member and its orientation. 
The same polluting (2\% random noise) and denoising procedure, explained earlier in section 4.1, is applied on simulated $\left\{\bar{\varepsilon}_{t}^{j}\right\}$. For each member, the symbol space was generated and then the damage localization procedure was performed by developing anomaly measures of different elements. By comparing the anomaly measures of different members, it is possible to identify the location of damage.

Table 2. Introduced damage cases for the truss structure

\begin{tabular}{|c|c|c|}
\hline & Single state damage & Multiple state damage \\
\hline Damage cases & $\begin{array}{l}\text { Element } 24 \\
\text { Element } 7 \\
\text { Element } 8 \\
\text { Element } 11 \\
\text { Element } 16 \\
\text { Element } 22\end{array}$ & $\begin{array}{l}\text { Elements } 7 \text { and } 8 \\
\text { Elements } 11 \text { and } 16 \\
\text { Elements } 13 \text { and } 19 \\
\text { Elements } 14 \text { and } 22\end{array}$ \\
\hline
\end{tabular}

The damage localization results are, respectively, presented in Fig. 12 and Fig. 13 for single state and multiple state damages. In all considered damage scenarios the maximum value of anomaly measure corresponds to the damaged location. By investigating Fig.12, it can be seen that in some graphs a false peak appears in an undamaged element; however, the magnitude of the false alarm is much smaller than the damage index corresponds to the damaged element. The interesting point lies in the fact that the obtained false alarms correspond to undamaged elements which are in the neighboring of damaged elements. For instance, in Fig.12 (b) false alarm is in undamaged element 19 which is located in adjacent of damaged element 7; the same finding can be seen in Fig. 12 (f) where the false peak happens in undamaged element 15 which is in neighboring of damaged element 22.

In the case of multiple state damage, Fig.13, all the damage cases can be reliably localized. Again, in some graphs a false peak appears which corresponds to undamaged element located in the neighboring of one of the real damages. For example, in Fig.13 (a), false peak is in undamaged element 13 which is exactly in the vicinity of damaged element 8 . As before, the damage index value which corresponds to the false peak is much smaller than the damage index related to the real damage.

Based on the obtained results, it can be seen that the location of deterioration can be accurately identified either in single or multiple state. Also, the findings demonstrate that the method is robust to the changes in the ambient excitation. As mentioned earlier, at each stage of deterioration the response of the structure is obtained under a new ambient excitation.

(a)

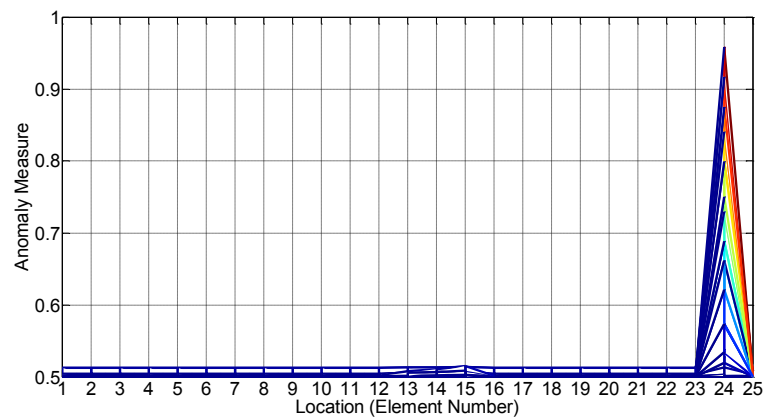

(c) (b)

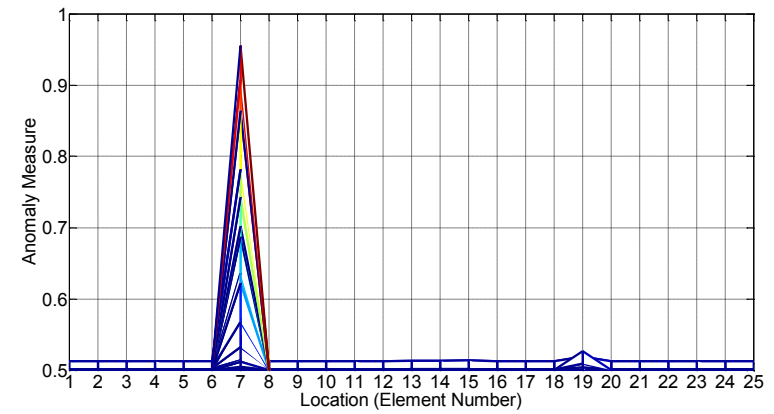

(d) 


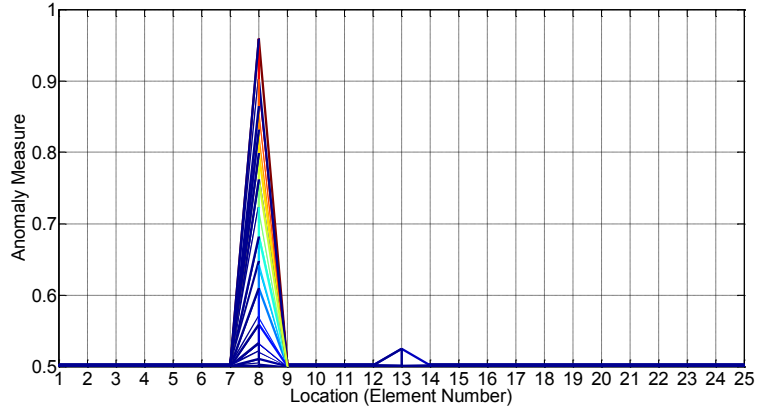

(e)

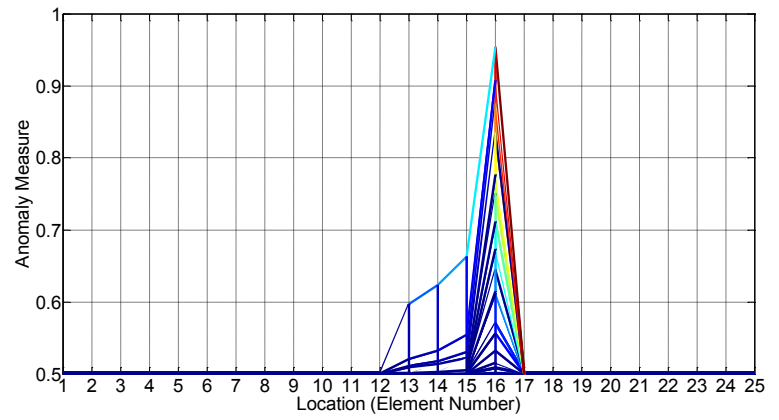

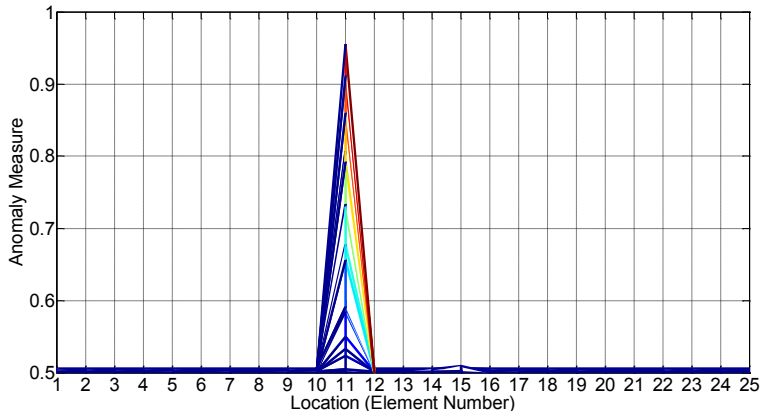

(f)

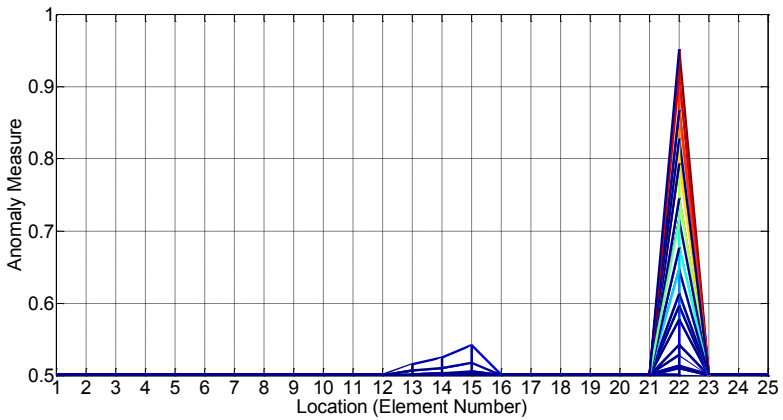

Figure 12. Damage localization results for single state damage at different locations, (a) damage in element 24, (b) damage in element 7, (c) damage in element 8, (d) damage in element 11, (e) damage in element 16, (f) damage in element 22.

(a)

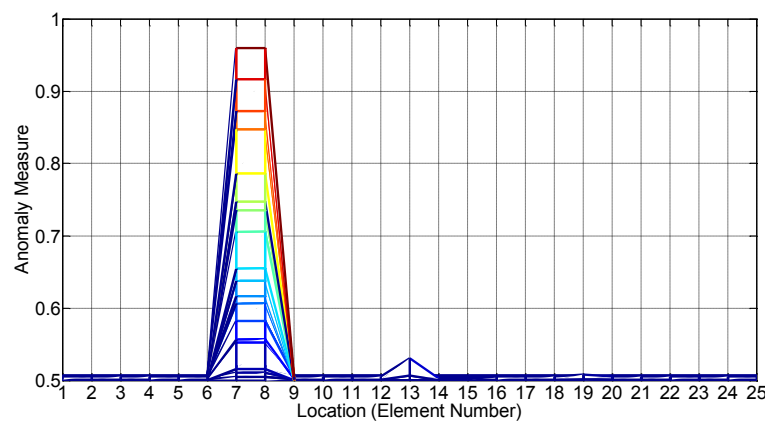

(c)

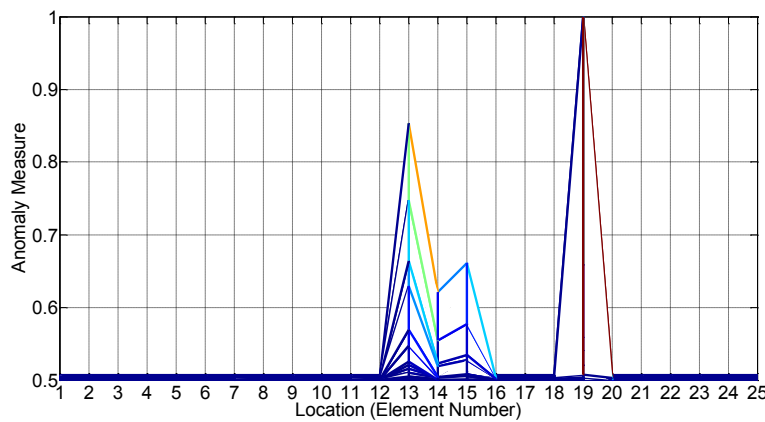

(b)

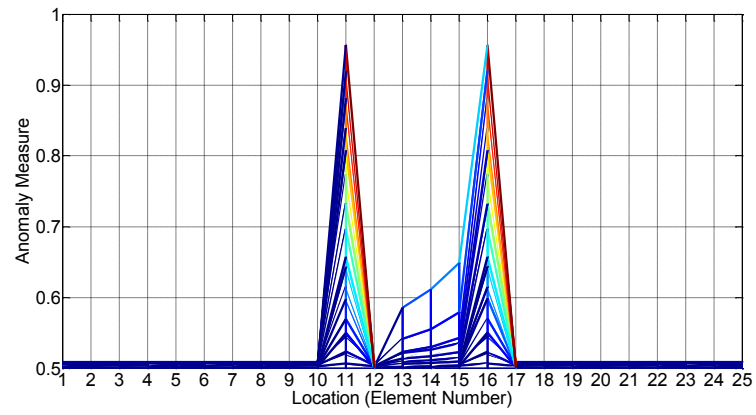

(d)

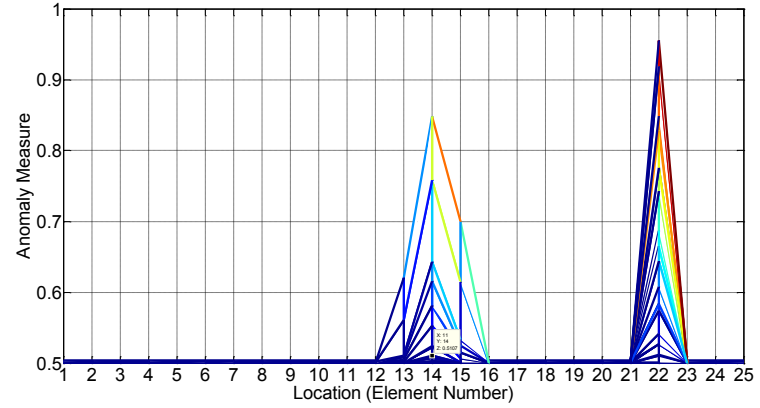

Figure 13. Damage localization results for multiple state damage at different locations, (a) damage in elements 7 and 8 (b) damage in elements 11 and 16, (c) damage in elements 13 and 19, (d) damage in elements 14 and 22. 


\section{Conclusions}

In the presented work, a new damage localization method was presented based on symbolic dynamics of time series data to detect and localize a gradually evolving deterioration in the system. Symbolic dynamics has some appealing features making it highly demanded for implementation in real-time observation application such as structural health monitoring. First, it significantly reduces the dimension of information and increase the efficiency of computations. Second, symbolic dynamics and the set of statistical measures built upon it represent a solid framework to address the main challenges of the analysis of non-stationary time data. Finally, STSA allows capturing the main features of the underlying system while alleviating the effects of harmful noise. The underlying concept behind the method is that the raw time-series measurements are uniquely mapped into a sequence of symbols and then statistical features of the symbols are used to describe the dynamic status of the system. The method does not need any prior knowledge of the structure's healthy state and the response of the structure in the current state can be used as the nominal state. The performance of the method was examined on a flexural beam and also a 2-D planar truss bridge subjected to varying Gaussian excitation in presence of $2 \%$ white noise in single and multiple damage states. Simulation results under various damage conditions confirmed the efficiency of the proposed approach for localization of gradually evolving deteriorations in the structure. In addition, the robustness of the method to the changes in the ambient excitation was demonstrated.

The authors would like to propose some suggestions for the future works:

1. First, the method needs to be verified by experimental data. The main challenge of the experiment is to create quantifiable gradual deterioration in the structure; in this regard, employing gradually added mass or using cyclic testing can be some alternatives.

2. In the presented work, only damage detection and localization schemes were studied but the authors believe that the method is capable of quantifying the damage. As explained earlier, for damages with the same severity but different locations, the method attributes different damage indices to the damages. This fact can be used for damage quantification as by knowing the damage location the damage index can be correlated to the damage severity by developing some geometry-dependent functions.

\section{Acknowledgement}

The authors wish to thank the Centre for Built Infrastructure Research (CBIR), University of Technology Sydney (UTS) for provision of financial support for this research work.

\section{References}

[1]. Sohn, Hoon, Charles R. Farrar, Francois M. Hemez, Devin D. Shunk, Daniel W. Stinemates, and Brett R. Nadler. (2003). A Review of Structural Health Monitoring Literature: 1996-2001. Los Alamos National Laboratory Report, LA-13976-MS.

[2]. Makki Alamdari, M., Li, J., \& Samali, B. (2014). FRF-based damage localization method with noise suppression approach. Journal of Sound and Vibration, 333(14), 3305-3320.

[3]. Baltazar, A., Hernandez-Salazar, C. D., \& Manzanares-Martinez, B. (2010). Study of wave propagation in a multiwire cable to determine structural damage. NDT \& E International, 43(8), 726-732.

[4]. Mata, J., Tavares de Castro, A., \& Sá da Costa, J. Time-frequency analysis for concrete dam safety control: Correlation between the daily variation of structural response and air temperature. Engineering Structures, (0).

[5]. Zhong, S. and Oyadiji, S. O. (2011), Crack detection in simply supported beams using stationary wavelet transform of modal data. Struct. Control Health Monit., 18: 169-190. doi: 10.1002/stc.366

[6]. Spanos, P. D., Failla, G., Santini, A. and Pappatico, M. (2006), Damage detection in Euler-Bernoulli beams via spatial wavelet analysis. Struct. Control Health Monit., 13: 472-487. doi: 10.1002/stc.118 
[7]. Ovanesova, A. V., \& Suárez, L. E. (2004). Applications of wavelet transforms to damage detection in frame structures. Engineering Structures, 26(1), 39-49.

[8]. Roveri, N., \& Carcaterra, A. (2012). Damage detection in structures under traveling loads by HilbertHuang transform. Mechanical Systems and Signal Processing, 28(0), 128-144.

[9]. Chen, H. G., Yan, Y. J., \& Jiang, J. S. (2007). Vibration-based damage detection in composite wingbox structures by HHT. Mechanical Systems and Signal Processing, 21(1), 307-321.

[10]. G.H. Dunteman. (1989). Principal Components Analysis, Sage Publications, London.

[11]. Bellino, A., Fasana, A., Garibaldi, L., \& Marchesiello, S. (2010). PCA-based detection of damage in timevarying systems. Mechanical Systems and Signal Processing, 24(7), 2250-2260.

[12]. Ni, Y. Q., Zhou, X. T., \& Ko, J. M. (2006). Experimental investigation of seismic damage identification using PCA-compressed frequency response functions and neural networks. Journal of Sound and Vibration, 290(1-2), 242-263.

[13]. Tibaduiza, D. A., Mujica, L. E. and Rodellar, J. (2013), Damage classification in structural health monitoring using principal component analysis and self-organizing maps. Struct. Control Health Monit., 20: 1303-1316. doi: 10.1002/stc.1540

[14]. Yang, Y., \& Nagarajaiah, S. Blind identification of damage in time-varying system using independent component analysis with wavelet transform. to appear in Mechanical Systems and Signal Processing.

[15]. Widodo, A., Yang, B., \& Han, T. (2007). Combination of independent component analysis and support vector machines for intelligent faults diagnosis of induction motors. Expert Systems with Applications, 32(2), 299-312.

[16]. Wang, Z., Chen, J., Dong, G., \& Zhou, Y. (2011). Constrained independent component analysis and its application to machine fault diagnosis. Mechanical Systems and Signal Processing, 25(7), 2501-2512.

[17]. Yuan, S., Wang, L., \& Peng, G. (2005). Neural network method based on a new damage signature for structural health monitoring. Thin-Walled Structures, 43(4), 553-563.

[18]. Zheng, S., Li, Z., \& Wang, H. (2011). A genetic fuzzy radial basis function neural network for structural health monitoring of composite laminated beams. Expert Systems with Applications, 38(9), 11837-11842.

[19]. Min, J., Park, S., Yun, C., Lee, C., \& Lee, C. (2012). Impedance-based structural health monitoring incorporating neural network technique for identification of damage type and severity. Engineering Structures, 39(0), 210-220.

[20]. Casciati, F. and Casciati, S. (2006), Structural health monitoring by Lyapunov exponents of non-linear time series. Struct. Control Health Monit., 13: 132-146. doi: 10.1002/stc.141

[21]. Adler, R.L., 1998. Symbolic dynamics and Markov partitions. Bulletin of the American Mathematical Society, New Series 35 (1), 1 / $/ 56$.

[22]. Brida, J. G., \& Punzo, L. F. (2003). Symbolic time series analysis and dynamic regimes. Structural Change and Economic Dynamics, 14(2), 159-183.

[23]. C. Rao, A. Ray, S. Sarkar, M. Yasar, Review and comparative evaluation of symbolic dynamic filtering for detection of anomaly patterns, 2008, doi: 10.1007/s11760-008-0061-8.

[24]. Graben, P. B. (2001). Estimating and improving the signal-to-noise ratio of time series by symbolic dynamics, Phys. Rev. E 64051104.

[25]. Li, R., Mita, A. and Zhou, J. (2013), Symbolization-based differential evolution strategy for identification of structural parameters. Struct. Control Health Monit., 20: 1255-1270. doi: 10.1002/stc.1530

[26]. Daw, C.S. \& Finney, C.E.A. (2002), A review of symbolic analysis of experimental data, College of William and Mary, Williamsburg, Virginia 23187-8795.

[27]. P. beim Graben, Phys. Rev. E 64, 051104 (2001).

[28]. Rajagopalan, V., Ray, A., Samsi, R., \& Mayer, J. (2007). Pattern identification in dynamical systems via symbolic time series analysis. Pattern Recognition, 40(11), 2897-2907.

[29]. Roveri, N., \& Carcaterra, A. (2012). Damage detection in structures under traveling loads by HilbertHuang transform. Mechanical Systems and Signal Processing, 28(0), 128-144.

[30]. Kunwar, A., Jha, R., Whelan, M., \& Janoyan, K. (2013). Damage detection in an experimental bridge model using hilbert?huang transform of transient vibrations. Structural Control and Health Monitoring, 20(1), 1-15.

[31]. P. beim Graben, J. D. Saddy, M. Schlesewsky, adn J.Kurths, Phys. Rev. E 62, 5518 (2000).

[32]. Cammarota, C., \& Rogora, E. (2005). Independence and symbolic independence of nonstationary heartbeat series during atrial fibrillation. Physica A: Statistical Mechanics and its Applications, 353(0), 323-335.

[33]. Arroyo, D., Latorre, R., Varona, P., \& Rodríguez, F. B. (2013). Application of symbolic dynamics to characterize coordinated activity in the context of biological neural networks. Journal of the Franklin Institute, 350(10), 2967-2981.

[34]. Shannon, Claude E. (1948). A Mathematical Theory of Communication. Bell System Technical Journal, 27 (3), 379-423. 
[35]. Ebeling, W., Nicolis, G., (1991). Entropy of symbolic sequences: the role of correlations. Europhysics Letters 14, 191-196.

[36]. Lang, M., Guo, H. , Odegard, J.E., Burrus, C.S., Wells, R.O., (1996). Noise reduction using an undecimated discrete wavelet transform, IEEE Signal Processing Letters 3 (1) 10-12.

[37]. Jiang, X., Mahadevan, S. and Adeli, H. (2007), Bayesian wavelet packet denoising for structural system identification. Struct. Control Health Monit., 14: 333-356. doi: 10.1002/stc.161

[38]. Ferreira da Silva, A. R. (2005). Wavelet denoising with evolutionary algorithms. Digital Signal Processing, 15(4), 382-399.

[39]. Pasti, L., Walczak, B., Massart, D. L., \& Reschiglian, P. (1999). Optimization of signal denoising in discrete wavelet transform. Chemometrics and Intelligent Laboratory Systems, 48(1), 21-34.

[40]. Narasimhan, S. V., Basumallick, N., \& Veena, S. (2012). Introduction to wavelet transform: A signal processing approach. Oxford: Alpha Science International Ltd.

[41]. D. Donoho, De-noising by soft-thresholding, IEEE Trans. Inform. Theory 41 (1995) 613-627.

[42]. Wang, P. C., Jeng, S. M., \& Yang, J. -. (1995). Characterization and modeling of stiffness reduction in SCS-6-ti composites under low cycle fatigue loading. Materials Science and Engineering: A, 200(1-2), 173-180.

[43]. Litewka, A. (1986). On stiffness and strength reduction of solids due to crack development. Engineering Fracture Mechanics, 25(5-6), 637-643.

[44]. Dharmaraju, N., Tiwari, R., \& Talukdar, S. (2005). Development of a novel hybrid reduction scheme for identification of an open crack model in a beam. Mechanical Systems and Signal Processing, 19(3), 633657.

[45]. Gaul,H., Weber,G., Rethmeier,M. (2011). Evaluation of fatigue crack propagation in spot welded joints by stiffness measurements. Int.J.Fatigue, 33(5), 740-745.

[46]. Oh, C. (2001). Application of wavelet transform in fatigue history editing. International Journal of Fatigue, 23(3), 241-250.

[47]. Mittermayr, C. R., Nikolov, S. G., Hutter, H., \& Grasserbauer, M. (1996). Wavelet denoising of Gaussian peaks: A comparative study. Chemometrics and Intelligent Laboratory Systems, 34(2), 187-202.

[48]. Mahbubur Rahman, S. M., \& Kamrul Hasan, M. (2003). Wavelet-domain iterative center weighted median filter for image denoising. Signal Processing, 83(5), 1001-1012.

[49]. Cho, D., \& Bui, T. D. (2005). Multivariate statistical modeling for image denoising using wavelet transforms. Signal Processing: Image Communication, 20(1), 77-89. 
Table 1. Introduced damage cases for the flexural beam

\begin{tabular}{l|l|l}
\hline \multicolumn{1}{c|}{ Damage cases } & \multicolumn{1}{c}{ C-F beam } & \multicolumn{1}{c}{ C-C beam } \\
\hline \multirow{3}{*}{ Single state damage } & $\begin{array}{l}\text { Element } 3 \\
\text { Element } 11 \\
\text { Element } 18\end{array}$ & $\begin{array}{l}\text { Element } 11 \\
\text { Element } 17\end{array}$ \\
\hline \multirow{3}{*}{ Multiple state damage } & $\begin{array}{l}\text { Elements } 3 \text { and } 11 \\
\text { Elements } 3 \text { and } 18\end{array}$ & $\begin{array}{l}\text { Elements } 11 \text { and } 17 \\
\text { Elements } 3 \text { and } 17\end{array}$ \\
& Elements 11 and 18 &
\end{tabular}

Table 2. Introduced damage cases for the truss structure

\begin{tabular}{l|l|l}
\hline & \multicolumn{1}{|c|}{ Single state damage } & \multicolumn{1}{c}{ Multiple state damage } \\
\hline & Element 24 & \\
& Element 7 & Elements 7 and 8 \\
Damage cases & Element 8 & Elements 11 and 16 \\
& Element 11 & Elements 13 and 19 \\
& Element 16 & Elements 14 and 22 \\
& Element 22 &
\end{tabular}

